\title{
Conclusions
}

10 A une température donnée (15 $5^{\circ} \mathrm{C}$. par exemple), il existe entre le taux de mouillage $T$ d'un lait quelconque, sa densité $D$, sa teneur en matière grasse $G$ (en grammes par litre), la densité $\rho$ de la matière grasse et la densité $\delta$ du lait écrémé non mouillé, la relation générale :

$$
T=\frac{1.000(\delta-D)-\left(\frac{\delta}{\rho}-1\right) G}{10(\delta-1)}
$$

( $T=$ nombre de centimètres eubes d'eau dans $100 \mathrm{~cm}^{3}$ de lait mouillé) ;

$2^{\circ}$ Pour le lait de vache (normal), à partir de la formule précédente, en donnant à $\rho$ et $\delta$ les valeurs minimum $\rho=0,91$ et $\delta=1,033$, on peut calculer immédiatement un taux minimum de mouillage : $\mathrm{T}_{\mathrm{m}}<\mathrm{T}$ :

$$
\mathrm{T}_{\mathrm{m}}=3(1.033-1.000 \mathrm{D})-0,4 \mathrm{G}
$$

L'approximation de cette formule est identique à celle que l'on obtient classiquement en calculant le mouillage à l'aide de la formule de Fleischmann (ou des formules similaires). Dans les conditions pratiques habituelles, cette formule sera suffisante, l'erreur absolue commise sur $\mathbf{T}_{\mathrm{m}}$ étant toujours inférieure à 5 ;

$3^{\circ}$ Enfin, pour un lait quelconque, à une température donnée, il existe entre la densité $\eta$ de l'extrait dégraissé, la densité $\delta$ de ce lait après écrémage et l'extrait dégraissé rectifié $\varepsilon$ (par litre de lait écrémé), la relation générale :

$$
1.000 \eta(\delta-1)=\varepsilon(\eta-1)
$$

permettant de calculer l'un de ces' éléments, connaissant les deux autres.

(Laboratoire de Chimie de l'Ecolè Nationale Vétérinaire d'Alfort.)

\section{LA FLUORESCENCE DU LAIT}

$$
\text { par }
$$

MAURICE DÉRIBÉRÉ

Ingénieur E. B. P.

\section{Les phénomènes de fluorescence}

Parmi les méthodes d'essai physique appliquées aux contrôles chimiques et aux expertises, l'emploi de la lumière de Wood et des phénomènes de fluorescence a pris, depuis quelques années, un essor largement justifié par l'intérêt et l'importance des résultats obtenus. 
Nous rappellerons que la fluorescence est un phénomène particulier de photo-luminescence. Une substance douée de cette propriété et placée dans le champ obscur d'un rayonnement ultraviolet réfléchit les rayons aveq une longueur d'onde supérieure. Si l'émission est encore dans l'ultra-violet, la substance reste sombre et l'étude de cette fluorescence doit être faite par voie spectrographique. L'émission peut aussi être, de l'autre côté du visible, dans l'infra-rouge et l'on a alors les fluorescences infra-rouge ; mais si l'émission tombe dans le spectre visible l'objet s'illumine dans, l'obscurité en une couleur ou selon un spectre qui sont fonction de la nature de l'objet et de la présence d'impuretés luminogènes.

L'excitation n'est pas non plus à négliger. Certaines substances comme le verre, dont la fluorescence n'est pas excitée sous les rayons ultra-violets proches du visible (lumière de Wood) deviennent lumineux lorsqu ils sont irradiés par des rayons plus courts (ultraviolet lointain, rayons $X$, rayons eathodiques).

Le phénomène de fluorescence est, en réalité, très général, et la fluorescence peut déjà se produire dans le spectre visible : ainsi le platinocyanure de baryum qui réfléchit bien les couleurs rouge, orange, jaune et vert, selon les lois habituelles, réfléchit par contre le bleu et le violet, tout comme l'ultra-violet, en vert. Cette substance est donc dotée d'une fluoreseence verte et l'on peut dire que cette fluorescence est activable à partir de la raie chiffrant le bleu dans le langage des physiciens, soit $4.600 \mathrm{~A}^{\circ}\left(1 \mathrm{~A}^{0}=10-^{4}\right.$ microns ou $10-10$ mètres).

L'ultra-violet filtré et invisible est utilisé, en pratique, pour l'excitation des fluorescences, et l'on retient la zone voisine de $3.600 \mathrm{~A}^{\circ}$, c'est-à-dire le proche ultra-violet. Cette zone, en effet, est facile à obtenir et ne présente pas de danger, du point de vue physiologique, pour les expérimentateurs.

Pour faire des essais de fluorescence, il fallait produire les rayons ultra-violets. Pour cela, les lampes à are, les brûleurs à vapeurs de mercure, des lampes à filaments poussés et plus récemment des lampes ampoules à vapeur de mercure à haute pression donnèrent de bonnes solutions. Mais toutes ces sources comportent, avec l'ultra-violet, beaucoup de visible qu'il convient d'isoler pour faciliter les examens. Pour cela, WooD, physicien américain, réalisa un verre noir à l'oxyde de niekel ne laissant passer que les raies ultra-violettes de 3.650 à $3.663 \mathrm{~A}^{0}$ et la raie $3.341 \mathrm{~A}^{0}$ du mercure. Cette lumière filtrée prit le nom de lumière de Wood. Il existe aujourd'hui toute une gamme d'écrans genre Wood donnant des rayonnements isolés du même genre, plus ou moins restreints dans le spectre. 
Les appareils ont fait aussi de remarquables progrès et, sous plusieurs formes qui ont chacune leurs avantages et leurs inconvénients, ils mettent à la portée de nombreuses industries les investigations et contrôles en lumière de Wogd.

Ces applications sont devenues énormes en importance et en qualité. En effet, on conçoit aisément que, puisque chaque substance réagit différemment et réfléchit une couleur propre lorsqu'elle est placée dans les rayons ultra-violets obscurs et filtrés, il y a là une méthode d'analyse extrêmement pratique, simple et rapide.

Cependant, il ne faudra pas perdre de vue que la plupart des corps purs n'ont pas de fluorescence et demeurent obscurs sous la lampe, que des traces parfois minimes d'impuretés affectent et modifient la fluorescence - ce qui par ailleurs est souvent utilisable - que des modalités de fabrication peuvent intervenir aussi.

Des méthodes d'essai particulières ont souvent aussi leur raison d'être. C'est ainsi que, pour examiner un solide, il sera souvent bon de faire le contrôle sur une cassure récente ou sur poudre ; e'est ainsi que, pour examiner un mélange liquide, on obtiendra souvent d'excellents résultats en opérant des montées capillaires sur une bande de papier filtre qui montrera ensuite des auréoles à fluorescences caraetéristiques ; que des solutions de corps gras dans des solvants sont souvent plus intéressants à observer, etc.

Les techniques précises avec détermination de spectres de fluorescence complets sont encore plus précises. Nous avons aussi recommandé [1], dans l'examen des solutions aqueuses, d'opérer sur plaque à touche à neuf godets où sont réalisés les $p \mathrm{H}$ et $r \mathrm{H}$ extrêmes (ou au moins notables selon le schéma) :

\begin{tabular}{lll}
$\begin{array}{l}\text { acide et } \\
\text { oxydant }\end{array}$ & $\begin{array}{l}\text { neutre et } \\
\text { oxydant }\end{array}$ & $\begin{array}{l}\text { alcalin et } \\
\text { oxydant }\end{array}$ \\
\hline $\begin{array}{l}\text { acide et } \\
\text { indifférent }\end{array}$ & $\begin{array}{l}\text { neutre et } \\
\text { indifférent }\end{array}$ & $\begin{array}{l}\text { alcalin et } \\
\text { indifférent }\end{array}$ \\
\hline $\begin{array}{l}\text { acide et } \\
\text { réducteur }\end{array}$ & $\begin{array}{l}\text { neutre et } \\
\text { réducteur }\end{array}$ & $\begin{array}{l}\text { alcalin et } \\
\text { réducteur }\end{array}$
\end{tabular}

L'emploi de cette plaque a donné d'intéressants résultats dans l'essai des laits.

Nous n'insisterons pas ici sur les modes de production de la lumière de Wood ni sur les techniques d'emploi qui figurent en détail dans les traités spéciaux [2].

Nous ne passerons pas non plus en revue les domaines où les investigations en lumière de Wood sont fructueuses. Ceux-ci sont innombrables, tant dans les recherches chimiques pures que dans le contrôle des produits alimentaires, des fraudes et altérations les 
plus multiples. Les sels organiques, les antiseptiques, les colorants, les textiles, les huiles et graisses, les pigments... ont souvent des fluorescences caractéristiques ou donnent par des traitements simples des modifications type de cette luminescence. La plupart des industries trouvent ainsi dans cette méthode un auxiliaire précieux, souvent simplement additionnel, mais toujours utile s'il est bien manié et avec intelligence. Nous nous bornerons ici à examiner la fluorescence du lait.

\section{Comportement du lait de vache normal sous la lumière de Wood}

Si l'on place du lait de vache normal et frais sous les rayons invisibles de la lumière de Wood, on observe une fluorescence jaune canari très nette et earactéristique.

Si le lait est abandonné au repos, la teinte jaune originale de fluorescence se concentre dans la crème. Peu à peu on voit, par conséquence, la crème surnageante emmagasiner toute la lumineseence, avec un ton toutefois plus clair et d'autant plus pâle que le lait était plus chargé en matières grasses. Le reste du liquide perd progressivement sa fluorescence jaune pour devenir, sous les rayons fluorigènes, blanchâtre ou bleuâtre peu lumineux. Cette modification du jaune au blane bleuâtre se constate nettement au bout de 20 à 24 heures. Toutefois, elle ne se produit pas si, avant d'abandonner le lait au repos pour cet essai, on a pris la précaution. de le faire bouillir ou de le traiter par la formaline. En outre, si, ayant laissé du lait frais non bouilli dans un tube jusqu'à coloration bleu pâle (le jaune étant entièrement passé dans la crème surnageante), on agite vivement le liquide, on retrouve la fluorescence jaune pour l'émulsion ainsi reformée. Celle-ci vire à nouveau au bleu en moins d'une heure et l'essai peut être renouvelé plusieurs fois dans les mêmes conditions.

Ces faits, ainsi que la plupart de ceux que nous mentionnons plus loin, ont été observés par de nombreux auteurs qui ont étudié la fluorescence du lait [3 à 15].

Si les essais se font sous vide, on observe que le changement de la eoloration de fluorescence du jaune au blanc bleuâtre se fait plus lentement. Il semble donc qu'il n'y ait pas seulement concentration des principes gras qui fluorescent en jaune, dans la crème, mais aussi une destruction de la fluorescence jaune du petit-lait par oxydation. L'oxygène de l'air est ainsi un agent actif du virage de la fluorescence.

Ce virage peut également être provoqué par oxydation photochimique, e'est-à-dire par irradiation du lait sous les rayons ultraviolets. Le lait irradié, en effet, perd sa fluoreseence jaune pour 
prendre une fluorescence bleu. Cette modification de luminescence avait été signalée, dès 1927, par Gerngross et Sohulz [5]. Ces auteurs, en signalant que, par irradiation la fluorescence jaune canari du petit-lait frais virait très rapidement à un bleu azur lumineux, écrivaient qu' "il 's'agit là d'un des phénomènes les plus frappants et les plus beaux que nous ayons pu observer jusqu'à présent sous l'action des rayons ultra-violets de la lampe de quartz à analyse ".

Le chauffage du lait, jusqu'à la température d'ébullition, est sans effet appréciable sur la fluorescence. Par contre, uhe ébullition maintenue et prolongée fait passer la fluorescence au blanc bleuâtre. Il en est de même du chaufage poussé au-dessus du point d'ébullition; ainsi un lait porté à $130^{\circ}$ perd sa fluorescence jaune et fluoresce en bleu ou en blanc bleuâtre.

Par aeidification ou alealinisation, on peut aussi atténuer la fluorescence, mais celle-ci peut être alors rétablie par neutralisation.

Il est évident que ces diverses modalités de comportement du lait de vache normal sous la lumière de Wood peuvent être, très utilement, employées dans la pratique des contrôles. Un lait frais, normal, non écrémé, présentera une fluores cence jaune canari. Au repos, on pourra distinguer la concentratión de la crème, dont la fluorescence est plus claire, eependant que le lait écrémé, soit lors de cet abandon au repos, soit artificiellement avant livraison, sera d'un jaune terne, bien que plus net et moins blanc à l'état frais que la crème. frais.

Un lait qui fluoresce en bleu, blanc bleuâtre ou violacé n'est pas

Après caillage un lait tourné montre, en lumière de Wood, des particules jaunes dans le liquide blanc bleuté, s'il était frais. S'il 'était ancien, les particules jaunes n'existent pas et il est alors permis de dire que le lait a tourné parce qu'il n'était pas frais.

\section{Défauts et falsifications}

L'examen en lumière de Wood permet, en bien des cas, d'utiles constatations sur les défauts du lait, certaines falsifications, la présence d'impuretés, etc.

La présence d'impuretés telles que foin, pus, matières fécales... est nettement plus facile à observer sous la lumière de Wood que sous l'éclairage ordinaire. Dans le cas du pus, l'emploi de bandes de capillarité donne des anneaux bleuâtres caractéristiques en lumière de Wood.

Un lait de vache frais, chargé de streptocoques, présente une fluorescence nettement plus verdâtre que celle du lait sain. Dans 
cet essai, il sera utile de réaliser des gammes de comparaison avec des matières fluorescentes ou des solutions fluorescentes que l'on conservera en boîtes fermées à l'abri de la lumière et qui seront remplacées, par exemple, tous les deux ou trois mois.

Un lait additionné d'eau amidonnée montre également une fluores cence plus verdâtre et due à la fluorescence bleue de l'amidon. Après vieillissement, la fluorescence est d'un bleu plus prononcé et plus mat. La présence d'amidon, d'abord difficile, à observer en lumière de Wood dans un lait est, au contraire, bien caractéristique pour un observateur possédant une certaine pratique. A cèt égard, on pourra conseiller de se familiariser dans l'examen de eette, fraude en réalisant artificiellement des suspensions légères d'amidon et en les introduisant en proportions variées dans un lait frais.

Le lait additionné d'eau stérilisée est d'un jaune plus pâle que le lait non additionné. Le lait écrémé est, ainsi que nous l'avons déjà signalé, plus clair et plus terne que le lait non écrémé.

L'addition au lait de certains antiseptiques comme l'acide salicylique, l'abrastol, l'eau oxygénée donne au liquide une fluorescence qui varie sensiblement avec la nature et la quantité de l'addition. Par contre, d'autres incorporations comme celles d'acide borique, de formaline ou de bicarbonate de soude ne peuvent être décelées par fluorescence.

L'examen en lumière de Wood de laits antiseptisés à l'acide salicylique ou avec des composés de cet acide, tels que le salol..., est particulièrement intéressant et doit être retenu pour les contrôles de fraudes et falsifications.

L'acide salicylique et les salicylates ont, en effet, une fluorescence nette et distinctive. L'acide salicylique ou orthoxybenzoïque $\mathrm{C}^{7} \mathrm{H}^{6} \mathrm{O}^{3}$ possède une belle fluorescence violette blanchâtre qui permet de le distinguer de l'acide méta, doté lui-même d'une fluorescence de même ton, mais beaucoup moins vive, et de l'acide para, dont la fluorescence bleu violet est encore plus faible.

Les salicylates alcalins sont encore plus fluorescents et c'est avec le salicylate de sodium que l'on trouve la fluorescence la plus intense. Aussi, dans les essais, on ramènera les composés salicyliques au salicylate de soude en additionnant le lait de quelques gouttes de soude caustique $[16,17]$.

La fluorescence du salicylate de sodium est si vive qu'elle peut être détectée dans le lait à $1 / 25.000$ parties [6] et même, en se plaçant dans des conditions spéciales et favorables, à $1 / 50.000$ parties [18]. On peut constituer, pour les dosages, des gammes étalons de comparaison. 


\section{Eléments fluorescents du lait}

La fluorescence normale du lait est due à la présence de divers principes fluorescents dont les proportions relatives peuvent être influentes.

L'opinion la plus généralement admise jusqu'à ces dernières années était que la fluorescence du lait était principalement due à des pigments végétaux albumineux.

A. Desmoulik̀res et E. Gautrelet avaient indiqué, dès 1903, que le lait pouvait contenir de l'urobiline. Or, il est connu que l'urobiline donne,e, en présence de traces de sel de zine, ou même pure (mais à moindre degré) une fluorescence verte. L'intensité optimum de cette fluorescence se place au $p$ H 7 à 7,2 .

La présence de coproporphyrine (fluorescence rougeâtre), de caroténoïdes et de vitamine A est aussi connue et ces éléments peuvent jouer sur la fluorescence.

Cependant, il est aujourd'hui établi que la fluorescence normale du lait (petit-lait et lactosérum), de couleur jaune à jaune vert, est principalement due à la présence de la lactoflavine, ou vitamine B2.

L'importance, en ce sens, de la lactoflavine, nous impose, ici; quelques précisions.

Les flavines sont, comme l'on sait, des pigments azotés et colorés en jaune, d'où leur dénomination, proposée par KUHN en 1933, par comparaison avec le principe colorant de même nom. Les travaux d'une pleïade de chercheurs (Ellinger et Koschara, KUhN, KARRER...) ont montré qu'il existait des rapports étroits entre ces flavines, telles qu'on les trouve dans les tissus animaux, le lait, les oufs, le foie des vertébrés, la levure..., et la vitamine B2 d'une part, et, d'autre part, avec la "diastase jaune" ou ferment oxydo-reducteur, de Warburg et Christian. Les uns comme les autres ont, comme caractère physique en vue, identité d'une belle couleur jaune avec fluorescence verte lumineuse sous les rayons violets et ultra-violets, déjà observable au jour en certaines conditions favorables (notamment en lumière bleue et violette).

La lactoflavine, qui s'identifie bien avec la vitamine B2, est une ribo-flavine (flavine contenant du ribose, selon KARRER). sa constitution chimique est maintenant bien établie et a été démontrée par la synthèse. Il s'agit de substances à noyau d'isoalloxazine (à 4 atomes d'azote). La formule brute de la lacto- 
flavine, de poids moléculaire 376 , est $\mathrm{C}^{17} \mathrm{H}^{20} \mathrm{~N}^{4} \mathrm{O}^{6}$ et se développe (KARRER) :

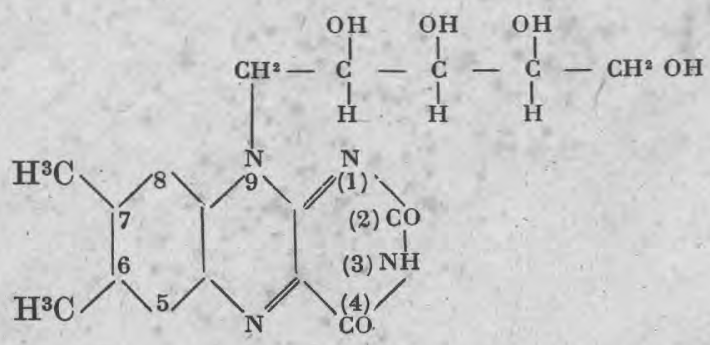

ou 6,7 - Diméthyl - 9 - (tétraoxypentyl) - iso-alloxazine ou $6-7$ Diméthyl $-9-\mathrm{d}-$ Ribo-flavine.

Comme le pigment jaune et comme toutes les flavines naturelles, la lactoflavine est très sensible à l'action de la lumière. La photolyse en milieu alcalin fournit le photo dérivé "lumiflavine" en même temps que du sucre sous forme pentose.

La lumiflavine est de même composition $\mathrm{C}^{13} \mathrm{H}^{12} \mathrm{~N}^{4} \mathrm{O}^{2}$ développable selon la forme :<smiles>CC1CC2NC(O)C(O)N(C)C2CC1C</smiles>

que le photodérivé provenant du pigment jaune irradié, e'est le 6-7-9 Triméthyl-isoalloxazine.

Par l'action de la potasse à chaud, la lumiflavine se décompose en urée et en acide oxocarbonique $\mathrm{C}^{12} \mathrm{H}^{12} \mathrm{~N}^{2} \mathrm{O}^{3}$.

Si l'irradiation, au lieu d'être faite en milieu alealin, est réalisée en solution neutre, il apparaît un dérivé nouveau soluble dans le chloroforme, et ne possédant plus la fluorescence verte, mais une fluorescence bleue. C'est le "luminochrome » identifié par KARRER et de formule $\mathrm{C}^{12} \mathrm{H}^{10} \mathrm{~N}^{4} \mathrm{O}^{2}$ développable selon le schéma :

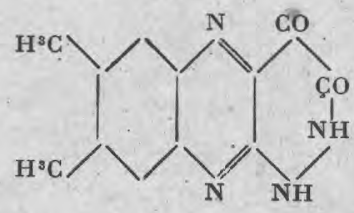

ou $6-7$ diméthyl-alloxazine. 
En définitive, la filiation chimique des flavines part de l'alloxazine et la genèse, à partir de l'alloxane est, selon BIERry et Gouzon, la suivante.<smiles></smiles>

o - Diamixobenzène + alloxane $\rightarrow$ alloxazine.

La flavine est, chimiquement, une iso-alloxazine. KUHN a toutefois fait remarquer qu'il serait plus correct de désigner la flavine sous le nom chimique de benz-iso-alloxazine. Le terme "flavine " possède l'avantage de la brièveté ; quant à la lactoflavine c'est, comme nous l'avons dit, la $6-7$ diméthyl - 9 tétraoxy - $\mathrm{n}$ - anyl - flavine :

La flavine est de forme (isoalloxazine) :

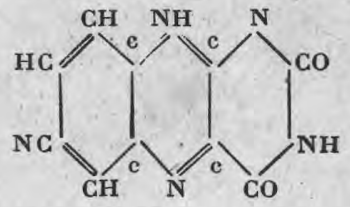

et le rattachement du dérivé lactoflavine sur cette base est simple, de même que le passage par irradiation de la lactoflavine à la lumiflavine en milieu alcalin :

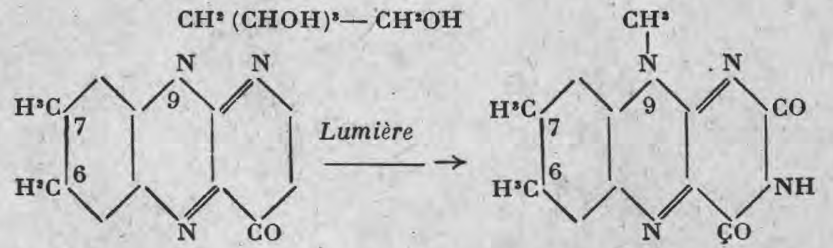

lactoflavine $\left(\mathrm{C}^{17} \mathrm{H}^{20} \mathrm{~N}^{4} \mathrm{O}^{6}\right) \rightarrow$ lumiflavine $\left(\mathrm{C}^{13} \mathrm{H}^{12} \mathrm{~N}^{4} \mathrm{O}^{2}\right)$ ou en lumichrome en milieu neutre :

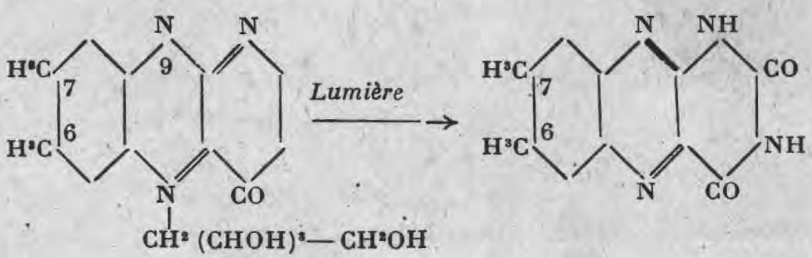

lactoflavine $\left(\mathrm{C}^{17} \mathrm{H}^{20} \mathrm{~N}^{4} \mathrm{O}^{6}\right) \longrightarrow$ lumichrome $\left(\mathrm{C}^{12} \mathrm{H}^{10} \mathrm{~N}^{4} \mathrm{O}^{2}\right)$. 
Lorsque la transformation par irradiation se fait en l'absence d'oxygène, la modification est moins profonde et la réaction plus difficile. Dans ces conditions, la lactoflavine fournit un dérivé incolore à partir duquel on peut obtenir, par action de l'air, la deuteroflavine de fluorescence verte [19].

La fluorescence de la lactoflavine en solution aqueuse neutre est forte et bien nette. La réaction du milieu est un facteur influent quant à son intensité, KUHN et MoRUzzI ont examiné cette intensité de fluorescence en fonction du $p \mathrm{H}$ pour la lactoflavine et la lumiflavine. L'optimum se prolonge en palier de $p H 4$ à 8 mais, de chaque côté de ce palier, il y a extinction assez rapide de la fluorescence [21].

Dans certaines conditions on peut observer un virage au bleu par alcalinisation.

KUHN a fait remarquer que, la flavine étant une substance amphotère, seule la molécule électriquement neutre est douée de fluorescence. Le cation incolore et l'anion jaune ne sont pas fluorescents ou le sont peu. Par contre, la fluorescence est au maximum dans la zone isoélectrique. Les modifications de fluorescence provoquées par le $p H$ sont réversibles si elles sont assez rapides pour que la molécule de base subsite.

La concentration des solutions est en règle générale influente sur les fluores cences.

KarRer et Fritzsche, en 1935, ont étudié [22] les variations d'intensité de fluoreseence de la lactoflavine et de diverses flavines synthétiques en fonction de la concentration des solutions. Pour la lactoflavine, les résultats obtenus (avec photomètre Leifo de Leitz et filtre) furent (excitation avec lumière de $5,320 \mathrm{~A}^{\circ}$ ) :

$\begin{array}{ccc}\begin{array}{c}\text { Concentration } \% \\ \left(\% \times 10^{-3}\right)\end{array} & \text { Division lue } & \begin{array}{c}\text { Intensité de fluorescence } \\ \text { en unités du photomètre }\end{array} \\ 12 & 10,75 & 34,8 \\ 6 & 14,3 & 61,0 \\ 3 & 16,1 & 76,9 \\ 2 & 15,5 & 71,4 \\ 1,5 & 14,5 & 62,7 \\ 1,2 & 13,7 & 56,1 \\ 1,0 & 13,3 & 52,9 \\ 0,6 & 10,8 & 35,1 \\ 0,54 & 10,5 & 33,2\end{array}$

Ceci montre un net maximum de fluorescence pour une concentration de $0,003 \%$, le $p H$ de la solution aqueuse considérée étant constant et fixé au $p \mathrm{H}=7$.

Il est aisé de rapprocher les caractères de fluorescence de. la lactoflavine de ce qui a été dit à propos de la fluorescence du lait de vache normal. 
L'examen des fluorescences des flavines est particulièrement redevable des méthodes de spectrographie, mais les examens simples en lumière de Wood sont également intéressants et il est possible, par emploi de filtres ou par réglage du $p \mathrm{H}$ de se débarrasser dans les essais des fluorescences parasitaires gênantes [20].

Selon KuHN et Kaltschmiтt [23], la lactoflavine du lait est facilement dialysable et n'est pas combinée aux albumines, mais elle préexiste sous forme libre en forte proportion $(90 \%)$. Elle n'est pas non plus combinée sous forme de l'ester phosphorique (pigment coloré de la diastase jaune). Par suite, en soumettant du lait frais écrémé ou son extrait dialysé, à l'action d'un champ électrique (220 volts, 10 milliamp., $p \mathrm{H}=7,2$, tamponné avec phosphate $\mathrm{N} / 15$ ), la substance colorante à fluorescence verte ne subit pas de migration.

Cet état de liberté implique une facilité de dosage par fluorométrie.

Il impliquait aussi le rôle probable et aujourd'hui démontré de la fluorescence des flavines incluses sur la fluoreseence du lait et des produits laitiers.

C'est seulement en 1933-1934 que ce rôle fut indiqué et précisé. COHEN [24] montra ce point en 1934 tandis que RADLEY [13] établissait que la fluorescence bleue résultant de l'irradiation était due au lactochrome et à sa réduction en deux phases.

\section{Autres Iaits}

Le, comportement des différents laits n'est pas toujours le même que celui du lait de vache.

Gerngross et Schulz [5] ont indiqué que le lait frais de brebis ou de chèvre avaient, comme le lait de vache, une fluorescence jaune canari passant au bleu par irradiation. Par contre, ces fluorescences ne sont presque pas visibles pour le lait de femme qui est faiblement jaunâtre et passe très vite au bleuâtre. Il en est de même du lait de chienne.

Le lait de jument, de lionne, d'ânesse... a, même frais, une légère fluorescence bleuâtre.

JoACHINowits, en 1929, a fait les mêmes constatations.

La fluoreseence signalée pour le lait de vache existe en général, plus ou moins accentuée, chez les herbivores, mais elle est nulle ou très faible chez les carnivores.

C'est done dans l'examen des laits d'animaux herbivores que l'emploi des phénomènes de luminescence trouve sa meilleure signification et sa plus grande utilité pratique. 


\section{RÉFERENCES BIBLIOGRAPHIQUES}

[1] M. Déribérê. L'importance conjuguée du $p \mathrm{H}$ et du $r \mathrm{H}$ sur les phénomènes de fluorescence. Docum. Scient., septembre-octobre 1937.

[2] M. Déribéré. Les applications pratiques de la luminescence : fluorescence, phosphorescence, lumière noire. Préface de Max Haitinger. Un vol., Paris, Dunod, 1938. - G. Bernherm et M. GuYot. Traité d'analyse par les rayons ultra-violets filtrés. Paris, Maloine, 1932. J. Seyewetz. Les nouvelles méthodes d'analyse ; la lumière de Wood. Paris, Baillière, 1934. - Ch. DHéré. La fluorescence en biochimie. Paris, Presses Universitaires, 1937. - M. Dértbéré. Les applications de la lumière de Wood et des rayons ultra-violets dans les industries textiles et tinctoriales. Paris, E'ditions Textiles, 1937. - P. W. Danckwortw. Lumineszenz analyse. Leipzig, 1935, M. Haitinger. Die Fluoreszenzanalyse in der mikrochemie. Vienne, 1937. - M. Haitinger. Die Methoden der fluoreszenz mikroskopie. Vienne, 1934. - J. A. RADLey et J. Grant. Fluorescence analysis in ultra-violet light, Londres, 1935.

[3] Dr M. Champeil. La lumière de Wood. Thèse, Alger, 1925.

[4] T. Baumgartel. Analyse par luminescence des produits du lait et des fromages. Molkerei Zeitung, $\mathrm{n}^{\circ} 45, \mathrm{p} .141,1931$.

[5] O. Gerngross et M. Schultz. Sur la fluorescence du lait sous les rayons ultra-violets filtrés. Milchwirtsch. Forsch., no 6, p. 567, 1928 et Chem. Zeit., no 51, p. 501, 1927.

[6]. Y. Volmar. Utilisation des phénomènes de fluorescence dans l'analyse des substances alimentaires. Journ. Pharm. Chim., no 5, p. 435, 1927.

[7] K. SCHUtzLer. Analyse aux rayons ultra-violets dans l'industrie laitière. Milchwirtsch. Forsch., t. IV, p. 352, 1927.

[8] A. K. Anderson et H, O. Triebold. Etude biochimique du lait irradié. Journal Dairy Sc., t. VI, p. 469, novembre 1932.

[9] K. Braunsdorf. Luminescence de la crème en lumière ultra-violette. Zeits. Unters. Lebensmitt., t. LXIII, n 3, p. 308, mars 1932.

[10] G. W. Baker et S. TAUBeS. Lait et beurre : fluorescence. The Analyst. t. LVII, p. 375, 1932." "

[11] M. Haitinger. Sur le comportement de quelques produits de l'agrieulture en lumière ultra-violette. Fortschritte der Landwirtschaft, no 10 , 15 mai 1928.

[12] M. Déribérḱ. L'examen du lait de vache sous la lumière de Wood. Ann. Hygiène Publ., mars 1936.

[13] J. A. Radley. La fluorescence du lait et des produits de laiterie. The Analyst., t. LVIII, no 590, p. 527, septembre 1933.

[14] J. SpolverinI. La fluorescence du lait. Riv. italiana di Actinologia, t. II, p. 1, 1927.

[15] K. WURSTER. Sur l'emploi de la fluorescence du lait de vache en lumière ultra-violette filtrée. Milchwirtsch. Forsch, t. XI, p. 379, 1931.

[16] M. Déribéré, La fluorescence de l'acide salicylique. An. d'Hyg. Publ., no 8, p. $377,1937$.

[17] M. Déribéré. Contrôle par fluorescence des composés de l'acide salícylique. Ann. Ohim. Anal., t. XXIII, no 4, p. 89, 15 avril 1941. 
[18] J. A, Radley et J. Grant. Fluorescence analysis..., loc. cit. 2.

[19] M. Déribéré. La fluorescence des flavines. Bull. Assoc. Chimistes, no 1 , janvier 1939.

[20] M. Déribéré. A propos de la fluorescence des flavines. Bull. Assoc. Chimistes, $\mathrm{n}^{\circ}$ 6, juin 1939.

[21] R. KUнN et G. Moruzzi. Sur la constante de dissociation des flavines; effet du $p \mathrm{H}$ sur la fluorescence. Ber. d. Deutsch. Ohem. Gesel., t. LXVII p. $888,1934$.

[22] P. Karrer et H. Fritzsche, Courbes de fluorescence de la lactoflavine et de la flavine synthétique. Helv. Chimica Acta, t. XVIII, p, 911, juillet 1935 .

[23] R. KuHn et H. Kaltschmits. Sur l'état de la vitamine B2 dans le lait de vache. Ber, $d$, Deutsch. Chem. Gesel., t. LXVIII, p. 386, 6 février 1935 .

[24] J. Cohen. Acta Brev. Néerl., t. IV, p. 46, 1934.

\section{LE LAIT MALSAIN (1)}

par

\section{A. HOUDINIÈRE}

\section{GENERALITÉS SUR LES LAITS MALSAINS}

Il faut entendre par lait malsain un lait nuisible à la santé de l'homme ou des animaux.

Parmi les causes d'origine intra-vitam qui donnent au lait des propriétés malsaines, il convient de distinguer deux groupes :

\section{A. Les facteurs physiologiques.}

Chez les animaux en bonne santé, ils correspondent à certains états de la vie pendant lesquels le lait possède une composition ou des propriétés anormales.

Tels sont les états de rut, de gestation avancée, de parturition récente.

\section{B. Les facteurs pathologiques.}

Ce sont toutes les causes morbides ayant une répercussion directe ou indirecte sur la sécrétion lactée.

Tels sont, au premier ehef, les agents des maladies de la mamelle.

Viennent ensuite les responsables des affections dans lesquelles les grandes fonctions sont troublées.

Mais tous ces facteurs sont nombreux et variés. Ils vont du simple trouble fonctionnel (nymphomanie, surmenage) à l'utravirus (fièvre aphteuse) en passant, par le déséquilibre alimentaire,

(1) Rapport présenté à la Société de Pathologie comparée, Paris, séance du 13 janvier 1942. 